\title{
THE TREATMENT OF ASTHMA.
}

By GEORGE W. BRAY, M.B., M.R.C.P.

(Physician-in-Charge of Children's Department, Prince of Wales General Hospital, Tottenham; Assistant Physician, Princess Elizabeth of York Hospital for Children, Shadwell; Clinical Assistant, Asthma Clinic, Guy's Hospital; Late Asthma Research Scholar, The Hospital for Sick Children, Great Ormond Street.)

\section{Nature of Asthma.}

In using the term "asthma" I refer to a type of paroxysmal dyspnœa, principally expiratory in character, brought about by a spasmodic constriction of the bronchial muscle and/or the mechanical obstruction produced by swelling of or a thick viscid exudate from the bronchial mucous membrane. This type of difficulty in breathing has several other characteristics. It is frequently accompanied either in the past or present by such other allergic conditions as hay-fever, vasomotor rhinitis, eczema, urticaria or migraine in the individual sufferer or in other members of the family. The attack is generally excited by an abnormal hypersensitivity to some foreign substance; associated with an eosinophilia both at the site of the reaction and in the blood; and relieved immediately by the hypodermic injection of adrenaline. Such a definition excludes the inspiratory difficulty associated with cardiac or renal disease, and the respiratory embarrassment associated with bronchitis, thymic enlargement, congenital laryngeal stridor, laryngismus stridulus, retropharyngeal abscess and enlarged lymphatic glands in children. In adults it excludes the difficult breathing associated with chronic pulmonary tuberculosis, pulmonary new-growths, and pathological states of the pleura; bronchitis; foreign body in the lung and aneurism.

In taking the history of each individual case the facts elicited may be allocated to four groups, two related to the onset of the sensitization, namely, the allergic hereditary predisposition and/or tissue trauma; and two related to the production of the individual attack, namely, the specific sensitization and some non-specific catalyzing factor. These factors may be set out diagrammatically as follows:-

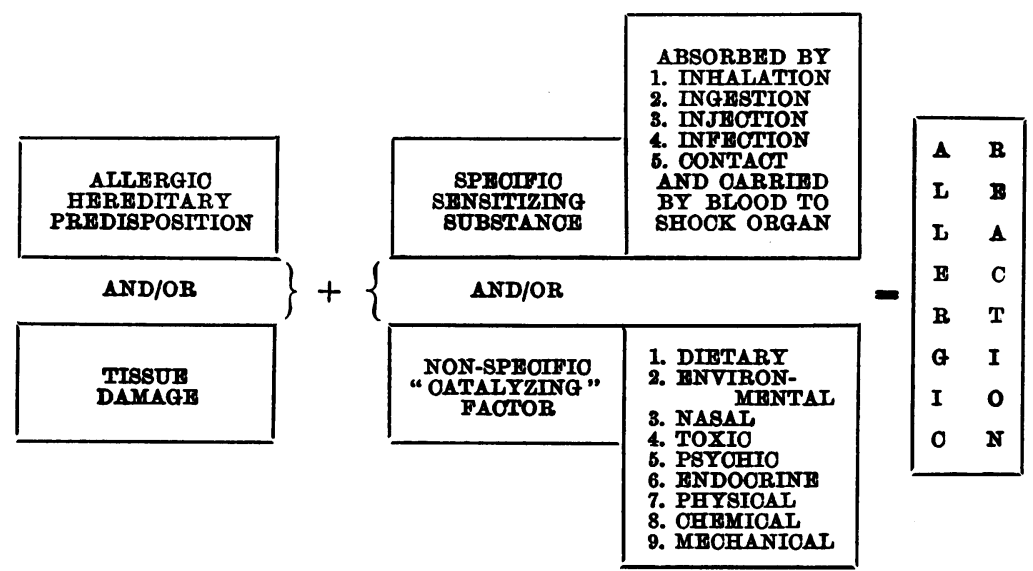

Illustrating the main factors concerned in the production of an allergic reaction.

It is only by keeping this diagram in mind when taking the history of the patient that the somewhat bewildering statements as to the cause of the attacks may be placed in their true categories and a rational method of treatment undertaken. 


\section{A. Factors related to the onset of sensitization.}

(1). The allergic hereditary predisposition. The tendency to develop allergic diseases runs predominantly in certain families who comprise from 2 to ro per cent. of the population of some countries. Enquiries should embrace all possible allergic manifestations and not only the one from which the patient under observation is suffering. Such a positive family history can be elicited in two out of every three cases seen. Whilst successful treatment may render a patient free from attacks there is at present no evidence that it affects the tendency to transmit the diathesis to further generations.

(2). Tissue Trauma. In a minority of cases, and especially those in which an hereditary tendency cannot be determined, a definite history of a severe illness shortly before the onset of the asthma can usually be obtained. In asthmatic children this has quite commonly been a pneumonic process complicating an infectious disease, such as whooping cough or measles; and in adults pneumonia, influenza or severe bronchitis play a similar part.

\section{B. Factors related to the production of the individual attack.}

(x). The specific sensitizing substance. The commonest sensitization is to some article of environment or diet, and the causative substances may be divided for convenience into five groups-

(i) Those absorbed by inhalation: Animal hairs, feathers, dusts, pollens moulds, fungi.

(ii) Those absorbed on ingestion: Foods, drugs.

(iii) Those absorbed from injection: Serums, drugs, bites and stings.

(iv) Those absorbed from infection: Bacteria, worms.

(v) Those absorbed through contact: Fabrics, chemical and physical agents.

In determining the specific cause or causes in each individual case a careful history, skin reactions, and elimination diets play a most important part. To those unaccustomed in dealing with these cases a few generalizations may be of assistance. Seldom does a food alone cause asthma unless the spasms had their onset during the first decade or are accompanied by skin lesions. In other words, in a pure uncomplicated case of asthma commencing after childhood, the attacks are more likely to be due to a sensitization to an inhaled substance than to a food. In a non-seasonal case the four commonest causes are feathers, animal hairs, dust and orris root, whilst in seasonal cases pollens must also be considered. Bacterial allergy plays very little part in the majority of cases; even in patients whose asthma always commences with a cold the bacteria merely lower the resistance of the mucous membranes of the respiratory tract and so make the absorption of the common inhalant sensitizing substances more readily brought about, especially if the patient is confined to the house or put to bed.

(2). The non-specific "catalyzing" factors. This motley group help to fire off the asthmatic spasm. In gross sensitivity each contact, however slight, leads to a reaction; but in less sensitive individuals the general tendency is for the body to avoid or overcome the ability to react. It is in this latter group with intervals 
of comparative freedom that other non-specific factors act. If, during exposure to the specific sensitizing substance, the resistance of the body is lowered by any of this group of psychic, endocrine, nasal, toxic, dietary or environmental factors a reaction will ensue. Hence they explain why a person only becomes asthmatic with a cold, during states of emotion or excitement, after exertion, following dietary indiscretions or in certain atmospheres.

\section{The Immediate Treatment of the Acute Attack.}

Inject subcutaneously in a child 2 minims, or in an adult 5 minims of a $I$ in a I,000 solution of adrenaline chloride. If the attack is a severe one it is always the best procedure to leave the needle in position after giving the first dose, for the effect of adrenaline is of short duration and smaller additional amounts are sure to be required to prevent the reappearance of the spasm. A I c.cm. Record syringe can be left filled with the adrenaline solution, and a relative be instructed to attach the syringe to the needle and give 2 minims of the solution at the end of one hour, a further two hours, four hours and eight hours, when as a rule the attack will be at an end and the patient have remained free from any recurrence in the meanwhile.

In "status asthmaticus" one or more minims of the adrenaline solution will need to be repeated more frequently-even as often as each minute-until relief of the spasm is obtained, and then each quarter of an hour for the first hour, each half-hour for two hours, each hour for three hours, and each two to four hours, until bedtime. If relief from adrenaline alone appears slower and less adequate an additional solution is necessary, made up as follows:

B.

Morphine Sulphate, $\frac{1}{4}$ grain.

Atropine Sulphate, I/Iooth grain.

Adrenaline Chloride (I/I,00o solution) to Io minims.

Continue the injections of plain adrenaline each minute, but each fifth minute inject one minim of this special solution instead, and the process is continued until the spasm is relieved or the Io minims of the solution have been injected. It is unwise to give more than 1 grain of morphia, even by this slow method; for, should the cough reflex be abolished and the spasm subside, large amounts of mucus will be poured out from the obstructed glands and unless this is expectorated the patient will be drowned in his own sputum. More patients die from the injudicious use of morphia than from the severity of the asthmatic spasm. Once the spasm has been relieved by these means the plain adrenaline solution is continued as above until the patient has been free for twenty-four hours. The maximum benefit from adrenaline is obtained when it is given at the earliest possible moment, in small doses, and repeated at gradually increasing intervals until the patient has been free for twenty-four hours.

Several additional measures are of great assistance in comforting the patient. Ephedrine hydrochloride should be given in $\frac{1}{2}-\frac{3}{4}$ grain doses each six hours, for several days. The body temperature should be restored by additional blankets and hot water bottles, as many patients are liable to develop a chill. If the patient is exhausted, stimulants are indicated and strong coffee in the morning or towards evening strong hot alcohol is indicated. Fluids and glucose are essential, for if the patient has had much adrenaline the liver supply of glycogen will have been depleted and the patient feel very worn out. The necessary sugar can be supplied either as frequent fruit drinks with glucose or as a glucose saline enema (3 ounces of glucose in a pint of normal saline). 
The use of adrenaline, ephedrine, atropine derivatives, cocaine or aromatic oils in sprays, or stramonium in the form of inhalations, powders, or cigarettes, $\stackrel{\leftarrow}{-}$ is to be deprecated. The relief afforded is only temporary, and they have anos extremely irritating effect on the bronchiolar mucous membrane; this, in turn,o increases the liability to further attacks. They tend to change an asthmatic with acute attacks and perfectly free intervals into a chronic, constantly wheezing indi- $\frac{\vec{\sigma}}{\overrightarrow{0}}$ vidual, though admittedly the spasms are less severe.

\section{The Intensive Treatment of the Asthmatic Sufferer.}

In order to prevent the recurrence of the asthmatic spasm it is necessary to $\vec{\omega}$ remove the specific causes or desensitize the patient to them; and to nullify theo various non-specific contributory factors that predispose the patient to his attacks.

\section{(x). The removal of the specific causes.}

\section{A. Inhalants.}

Most asthmatics, when properly tested with potent solutions by the intradermals method, will react to one or more of the feathers, dust, animal hair and orris root tests; so, as a general rule in any asthmatic it is wise to remove these as far as possible.

(a) BEDDING. Replace feather or down pillows, eiderdowns or bolsters, and feather, down, horsehair or rabbithair mattresses by a rubber mattress and pilloswew (Dunlopillo) and the best Java kapok bolster and eiderdown. All these bedding requisites can be supplied by Messrs. S. Brown and Son, ro, Houndsditch, E.C.3.ร

(b) PETs. Cats, dogs, horses and rabbits are common offenders, so should⿳亠丷⿵冂丶 be avoided as much as possible. Toys stuffed with animal hair should also be $ळ$ removed.

(c) Dust. This includes that encountered in the home, and in the course of various occupations. In the home there should be a minimum of curtains, ?: carpets, furniture and ledges or other dust-holding surfaces, and electric vacuumo cleaning is advisable. With the recent factory regulations the effect of occupa 3 tional dusts is not so pronounced.

(d) ORRIs ROOT. This forms the basis of most cosmetics such as face powder body powder, face creams and dry shampoos which form a very frequent cause of asthma and nasal catarrh, especially in women. Special orris-root-free cosmetics should always be used by women sufferers. (These are obtained from Messrs:o Boutalls, Ltd., Chemists, I50, Southampton Row, London, W.C.I, under the title of "Queen" products).

(e) Pollens. These play an important part in seasonal cases, especially the grasses in the summer.

\section{B. Foods.}

Usually the sufferer has learnt which foods affect him. If he gives a large list it is more probable that there is something wrong with his digestive powers than a specific sensitizing to a large number of foods. If the patient has no idea? 
what foods affect him, and all possibility of an inhalant sensitivity has been ruled out, then the easiest plan is to put him on a diet which avoids all the common causes of food allergy. The commonest reactions occur to-

(a) Cow's Milk. Fortunately in these cases it is the lactalbumen fraction, coagulable by heat, which is at fault, so well-boiled or heated milks as condensed or evaporated can be used. For infants a special half-cream, lactalbumen-free, acidified dried milk has been prepared (Allergilac, Cow and Gate, Ltd.). As the treatment of the milk destroys the vitamins these should be supplied as ostelin or radiostoleum in addition.

(b) EGG-WHITE. This is a frequent cause of asthma, especially in cases that have had eczema. Fortunately egg-yolk rarely causes trouble, and as this is the most nutritive part, it should be given.

(c) WhEAT. Cereal sensitivity depends to a large extent upon nationality, and wheaten foods are the staple articles in this country for porridge and bread. In asthmatic subjects this can be replaced by sago, tapioca or arrowroot as porridge, and by ryvita or oatmeal biscuits for bread.

(d) PIG. Pork, ham, bacon, lard and sausages or food containing them or cooked with them should be avoided.

(e) Other less common causes include Shellfish, Nuts, Cocoa and Chocolate, Strawberries, Beans and Peas, Potatoes and Tomatoes, Beef, APPLES AND BANANAS.

\section{(2). Desensitization.}

If the causes cannot be avoided or removed then desensitization is a necessary procedure. It may be brought about by specific or non-specific means, the former being the more usually successful.

(A) SPECIFIC. This is accomplished by mixing the specific substances to which the patient is sensitive into one solution and giving gradually increasing doses at daily, bi-weekly, or weekly intervals until the patient can tolerate the specific causes. If skin tests are not available to determine these causes or the facilities not at hand for preparing the special solution, a mixed solution of the common inhalant causes is the most reliable to use. A Mixed Inhalants Solution is prepared by Messrs. C. L. Bencard, Ltd., I52, Great Portland St., W.I, containing feathers, animal hairs, dust and orris root, and can be supplied with or without peptone, and with or without pollen. An intradermal test goes with the solution, and if a positive reaction is obtained the patient is sensitive to this group, and treatment with the solution will prove efficacious. Specific desensitization will occur to those members to which the patient is sensitive, whilst others will have a non-specific effect. Auto-hæmo-therapy-the withdrawal of ro c.c. of blood from the cubital vein and its immediate injection into the muscles of the buttocksometimes causes temporary relief.

(B) Non-Specific. Witte's peptone No. 30 is probably the best of the nonspecific measures, which include tuberculin, milk, "proteose" and vaccines. With regard to vaccines there is no appreciable difference in the results from stock or autogenous organisms, those from the sputum being commonly used. 


\section{(3). Medicaments.}

(A) Digestives. A large proportion of asthmatics, especially children who are more often food-sensitive than adults, show a deficient secretion of acid gastric juice on fractional gastric analysis. The majority also suffer from digestive symptoms, especially flatulence. In these cases the administration of hydrochloric acid by mouth immediately before or with meals is essential. This may be administered in solution as-

$\mathbf{B}$

Dilute Hydrochloric acid B.P. $\quad \ldots \quad \ldots \quad 30$ minims.

$\begin{array}{lllllll}\text { Dextrose } & \ldots & \ldots & \ldots & \ldots & \ldots & 30 \text { grains. }\end{array}$

Chloroform water $\ldots . . . \quad \ldots \quad \ldots$ to 2 drachms.

Two teaspoonfuls to be taken in a glass of orange or lemon drink, sweetened further if necessary, and taken immediately before or with each meal,

or in tablet form as-

Pulvis Acidus Tablets (Bencard)

One tablet placed on the tongue and swallowed with a wineglassful of water immediately before each meal.

(B) LINCTUs. The Chronic asthmatic needs a strong antispasmodic and sedas tive linctus at bedtime such as-

$\mathbf{B}$

$\begin{array}{llllll}\text { Pseudo-ephedrine } & \ldots & \ldots & \ldots & \ldots & \text { I grain. }\end{array}$

Tincture of Stramonium $\quad \ldots \quad \ldots \quad \ldots \quad$ I5 minims.

Syrup of Virginian Prune

Syrup of Codein Phosphate, of each ... to 2 drachms.

Two teaspoonfuls to be taken each bedtime for some months. bottles.)

(This is supplied as Linctus Anti-asthmatic by Bencard, Ltd., in 8 ounce

(c) ExPECTORANT. If the patient has much bronchitis an expectorant mixture may be necessary during the day, such as-

B

Iodide of Potassium ... $\quad \ldots \quad \ldots \quad \ldots \quad 4$ grains.

Tincture of Stramonium $\quad \ldots . \quad \ldots . \quad \ldots . \quad$ I5 minims.

Ethereal Tincture of Lobelia ... $\quad \ldots \quad \ldots \quad$ I5 minims.

Syrup of Virginian Prune $\quad \ldots \quad \ldots \quad \ldots \quad \ldots$ minims.

$\begin{array}{llllll}\text { Water } & \ldots & \ldots & \ldots & \ldots & \ldots\end{array}$ to 4 drachms.

One tablespoonful to be taken four-hourly during the day.

(4). The avoidance of contributory factors.

(A) BAtHs. A morning bath should be the rule. Hot baths towards bedtime predispose to attacks. 
(B) APERIENTS. A rapid morning saline purgative is preferable to the slowlyacting, over-night varieties which lead to hours of vagal irritation at the time the patient is most liable to attacks. A hot bath and a dose of syrup of figs at bedtime on a Friday night are the commonest predisposing causes to week-end asthma in school children.

(c) Diet. To prevent gastric distension, and so reflex vagal irritation, three small, easily-digested, simply-compounded meals a day are best; a good early breakfast, a hot substantial mid-day meat meal, and a light early supper several hours before retiring should be the rule. No food or drink should be taken after supper and especially not just before or after getting into bed.

(D) Reflex IrRitation. Besides from the bowel and stomach, reflexes may arise from the nose. Most nasal abnormalities in asthmatics are only coincidental or directly secondary to nasal allergic manifestations. Consequently, treatment should be conservative and non-surgical along allergic lines until as good results as possible are obtained. After the subsidence of the œdema, if the nasal pathology is still deterrent to adequate relief nasal surgery may be attempted. It should aim at freeing the nose from any source of infection, ensuring proper drainage and a free airway, as well as searching for any sensitive areas and removing tissue that may be responsible for reflex pressure symptoms.

(E) Psychical States. As the result of a specific sensitization the onset of attacks may become a conditioned reflex, which, after repeated exposures is capable of being fired off without the intervention of the specific sensitizing substance. When, as the result of desensitizing the patient against the primary cause of his reactions, he is shown he can experience exposure to the original cause without reaction all the psychical elements disappear, and especially the factor of expectation, and the case is solely an allergic one. This statement is borne out by the fact that it is possible to prepare some serum from a sufferer and inject it into a normal person's skin. If this site is tested a local reaction occurs only with the specific substance to which the patient was hypersensitive; hence an antisubstance circulates in the sufferer's blood. This fact is difficult to explain solely on a psychopathic basis.

(F) Endocrine Dysfunction. Statistics show that endocrine dysfunction is rare in cases of asthma, but there is no doubt that puberty, menstruation, marriage and the menopause play an important part in women sufferers.

(G) BREATHing Exercises. These should be of an expiratory nature, such as counting, humming, hissing and blowing, and should not be continued up to the point of fatigue. They teach the patient to deal with the expiratory difficulty of the asthmatic attack in the most efficient way; they tend to keep the chest and lungs of normal size; they help the thoracic muscles to maintain their natural postural length and tone; they teach the sufferer to use the lower part of his chest and diaphragm to a larger extent than he has been accustomed; and in established cases they help to overcome or prevent chest deformities. A manual of the exercises advised can be obtained from the Secretary, The Asthma Research Council, King's College, The Strand, W.C. (Price 2/-).

(н) Climate. The sufferer should be shielded from extremes of temperature, draughts, cold winds and fog. Asthmatics lose most of their symptoms at altitudes over 4,000 feet, whilst others become free at lower levels on entering a hospital, convalescent home, or spa. 